Pacific

Journal of

Mathematics

CONFORMAL METRICS WITH CONSTANT CURVATURE ONE AND FINITELY MANY CONICAL SINGULARITIES ON COMPACT RIEMANN SURFACES

Qing Chen, Wei Wang, Yingyi Wu And Bin Xu

Volume 273 No. 1

January 2015 


\title{
CONFORMAL METRICS WITH CONSTANT CURVATURE ONE AND FINITELY MANY CONICAL SINGULARITIES ON COMPACT RIEMANN SURFACES
}

\author{
Qing Chen, Wei Wang, Yingyi Wu And Bin Xu
}

\begin{abstract}
A conformal metric $g$ with constant curvature one and finitely many conical singularities on a compact Riemann surface $\Sigma$ can be thought of as the pullback of the standard metric on the 2-sphere by a multivalued locally univalent meromorphic function $f$ on $\Sigma \backslash$ \{singularities\}, called the developing map of the metric $g$. When the developing map $f$ of such a metric $g$ on the compact Riemann surface $\Sigma$ has reducible monodromy, we show that, up to some Möbius transformation on $f$, the logarithmic differential $d(\log f)$ of $f$ turns out to be an abelian differential of the third kind on $\Sigma$, which satisfies some properties and is called a character 1-form of $g$. Conversely given such an abelian differential $\omega$ of the third kind satisfying the above properties, we prove that there exists a unique 1-parameter family of conformal metrics on $\Sigma$ such that all these metrics have constant curvature one, the same conical singularities, and have $\omega$ as one of their character 1-forms. This provides new examples of conformal metrics on compact Riemann surfaces of constant curvature one and with singularities. Moreover we prove that the developing map is a rational function for a conformal metric $g$ with constant curvature one and finitely many conical singularities with angles in $2 \pi \mathbb{Z}_{>1}$ on the two-sphere.
\end{abstract}

\section{Introduction}

Let $\Sigma$ be a compact Riemann surface and $p$ a point on $\Sigma$. A conformal metric $g$ on $\Sigma$ has a conical singularity at $p$ with singular angle $2 \pi \alpha>0$ if in a neighborhood of $p$, $g=e^{2 \varphi}|d z|^{2}$, where $z$ is a local complex coordinate defined in the neighborhood of $p$ with $z(p)=0$ and $\varphi-(\alpha-1) \ln |z|$ is continuous in the neighborhood. Let

Chen is supported in part by the National Natural Science Foundation of China (grant no. 11271343). $\mathrm{Wu}$ is supported in part by the National Natural Science Foundation of China (grant no. 11071249) and the President Fund of UCAS. Xu is supported in part by Anhui Provincial Natural Science Foundation (grant no. 1208085MA01) and the Fundamental Research Funds for the Central Universities (grant no. WK0010000020).

MSC2010: primary 32Q30; secondary 34M35.

Keywords: conformal metric of constant curvature one, conical singularity, developing map, character 1-form. 
$p_{1}, \ldots, p_{n}$ be points of $\Sigma$ and $g$ a conformal metric on $\Sigma$ with conical singularity at $p_{j}$ of singular angle $2 \pi \alpha_{j}>0$ for $j=1, \ldots, n$. Then we say that the metric $g$ represents the divisor $D:=\sum_{j=1}^{n}\left(\alpha_{j}-1\right) P_{j}$. The Gauss-Bonnet formula says that the integral of the curvature on $\Sigma$ equals $2 \pi$ times

$$
\chi(\Sigma)+\operatorname{deg} D
$$

where $\chi(\Sigma)$ denotes the Euler number of $\Sigma$ and $\operatorname{deg} D=\sum_{j}\left(\alpha_{j}-1\right)$ the degree of the divisor $D$. A classical problem is whether there exists a conformal metric on $\Sigma$ of constant curvature $K$ representing the divisor $D$. If $K \leq 0$, then the unique metric exists if and only if the left-hand side $\chi(\Sigma)+\operatorname{deg} D \leq 0$; see [McOwen 1988; Troyanov 1991].

If $\chi(\Sigma)+\operatorname{deg} D>0$, or equivalently $K \equiv 1$ if we multiply the original metric by some constant, the problem turns to be quite subtle and is still open now, except that there are some partial results. Troyanov [1989] considered the case of two points on the sphere and proved that the necessary and sufficient condition in this case is $\alpha_{1}=\alpha_{2}$. A more general result also due to him [1991, Theorem 4] says that there exists a metric of constant positive curvature if

$$
0<\chi(\Sigma)+\operatorname{deg} D<\min \left\{2,2 \min \alpha_{j}\right\} .
$$

Luo and Tian [1992] proved that the above condition is also necessary and the metric is unique, provided that $\Sigma$ is the 2 -sphere and all angles lie in $(0,2 \pi)$. In case that $\Sigma$ is a sphere and the divisor $D$ is supported at three points, [Umehara and Yamada 2000; Eremenko 2004; Furuta and Hattori 1998; Fujimori et al. 2011] give a necessary and sufficient condition for the existence of the metric, which is also unique if and only if none of the three angles belongs to $2 \pi \mathbb{Z}_{>0}$.

We attack the problem by using the idea of a developing map, due to R. Bryant [1988, pp. 333-4], Umehara and Yamada [2000, p. 76] and Eremenko [2004, p. 3350]. Let $g$ be a conformal metric of constant curvature one on $\Sigma$ representing the divisor $D$. Let $\Sigma^{*}=\Sigma \backslash\left\{p_{1}, \ldots, p_{n}\right\}$. Every point $p$ in $\Sigma^{*}$ has a neighborhood $U_{p}$ isometric (so conformal) to an open set $\mathfrak{U}_{p}$ of the Riemann sphere $\overline{\mathbb{C}}$ endowed with the standard metric $g_{\mathrm{st}}$. Denoting by $\mathfrak{f}_{p}: U_{p} \rightarrow \mathfrak{U}_{p}$ this isometry (conformal map), Umehara and Yamada [2000] and Eremenko [2004] claimed that $\mathfrak{f}_{p}$ can be extended to the whole of $\Sigma^{*}$ by analytic continuation such that the extension gives a multivalued locally univalent meromorphic function $f$ on $\Sigma^{*}$, whose monodromy belongs to the group PSU(2) of orientation-preserving isometries of $\overline{\mathbb{C}}$ (see Lemma 2.1). Hence the metric $g$ can be thought of as the pullback

$$
g=\frac{4\left|f^{\prime}(z)\right|^{2}|d z|^{2}}{\left(1+|f(z)|^{2}\right)^{2}}
$$


under $f$ of $g_{\text {st }}$. Moreover, prompted by [Umehara and Yamada 2000, (2.10)] and [Eremenko 2004, (2)], we show in Lemma 3.1 that the Schwarzian $\{f, z\}$ of $f$ in a neighborhood $U_{j}$ of $p_{j}$ with complex coordinate $z$ with $z\left(p_{j}\right)=0$ has the form

$$
\{f, z\}:=\frac{f^{\prime \prime \prime}(z)}{f^{\prime}(z)}-\frac{3}{2}\left(\frac{f^{\prime \prime}(z)}{f^{\prime}(z)}\right)^{2}=\frac{c_{j}}{z^{2}}+\frac{d_{j}}{z}+\psi_{j}(z),
$$

where $c_{j}=\left(1-\alpha_{j}^{2}\right) / 2$, the $d_{j}$ are constants and the $\psi_{j}$ are holomorphic functions in $U_{j}$, dependent on the complex coordinate $z$. Since the value of $c_{j}$ is independent of the choice of the complex coordinate $z$, we say that $f$ is compatible with the divisor $D=\sum_{j}\left(\alpha_{j}-1\right) P_{j}$. We now arrive at:

Definition 1.1 [Umehara and Yamada 2000, p. 76]. Let $g$ be a conformal metric on $\Sigma$ of constant curvature one representing the divisor $D$. We call a multivalued locally univalent meromorphic function $f$ on $\Sigma^{*}$ a developing map of the metric $g$ if $g=f^{*} g_{\mathrm{st}}$.

On the other hand, if there exists a multivalued meromorphic function $f$ on $\Sigma^{*}$ which is compatible with the divisor $D$ and has monodromy in PSU(2), then there exists a conformal metric $g=f^{*} g_{\text {st }}$ with constant curvature one and representing $D$ (see Lemma 3.2). Therefore we can sum up the above into a necessary and sufficient condition (see Theorem 3.4) for the existence problem of conical conformal metrics of constant curvature one on $\Sigma$.

In this manuscript, we mainly focus on a special class of conical conformal metrics of constant curvature one, called reducible metrics, which we can classify by using abelian differentials of the third kind.

Definition 1.2 [Umehara and Yamada 2000, p. 76]. We call a conformal metric $g$ on $\Sigma$ of constant curvature one and with finitely many conical singularities an irreducible metric if the monodromy group of a developing map of the metric $g$ cannot be diagonalized, that is, the monodromy group has no fixed point on the Riemann sphere $\overline{\mathbb{C}}$ (see Lemma 4.1). We call $g$ reducible if the monodromy group has at least one fixed point on $\overline{\mathbb{C}}$. We call a reducible metric (non)trivial if the monodromy of a developing map of the metric is (non)trivial. Lemma 2.2 tells us that these definitions do not depend on the choice of a developing map.

A trivial reducible metric is a pullback of $g_{\text {st }}$ under some rational function on $\Sigma$ (Lemma 4.2). Each subgroup of PSU(2) having at least one fixed point on $\overline{\mathbb{C}}$ is abelian, and, up to conjugacy, can be thought of as a subgroup of the standard maximal torus

$$
\mathrm{U}(1)=\left\{\operatorname{diag}\left(e^{\sqrt{-1} \theta}, e^{-\sqrt{-1} \theta}\right) \mid \theta \in \mathbb{R}\right\} /\left\{ \pm I_{2}\right\}
$$


of PSU(2) (see Lemma 4.1). Each fractional transformation in U(1) is multiplication by $e^{2 \sqrt{-1} \theta}$. Therefore, for a nontrivial reducible metric $g$ on $\Sigma$, by Lemma 2.2 there exist exactly two developing maps, say $f$ and $1 / f$, of the metric $g$, whose monodromies belong to $\mathrm{U}(1)$.

Definition 1.3. Let $g$ be a nontrivial reducible metric on a compact Riemann surface $\Sigma$. We call a developing map $f$ of $g$ multiplicative if the monodromy of $f$ belongs to $\mathrm{U}(1)$. Such an $f$ is unique up to taking the reciprocal, and the logarithmic differential

$$
\omega:=d(\log f)=\frac{d f}{f}
$$

of the multiplicative developing map $f$ is a meromorphic 1-form on $\Sigma^{*}$. Actually, $\omega$ can be extended to be an abelian differential of the third kind on $\Sigma$ (see Lemma 4.3), which we call the character 1 -form of the reducible metric $g$. Hence the character 1 -form of a nontrivial reducible metric is unique up to sign.

Let $g$ be a trivial reducible metric on $\Sigma$. By Lemma 4.2, there exists a rational function $f: \Sigma \rightarrow \overline{\mathbb{C}}$ such that $g=f^{*} g_{\text {st }}$. By Lemma 2.2, each developing map of the metric $g$ is a rational function, and multiplicative. We call a character 1-form of the metric $g$ the logarithmic differential of a developing map of $g$. The character 1 -forms of the trivial reducible metric $g$ are automatically abelian differentials of the third kind on $\Sigma$.

To set up the notation for stating the properties of character 1-forms, we need say something more about the standard metric $g_{\text {st }}$ on the Riemann sphere $\overline{\mathbb{C}}$, which is a trivial reducible metric. The set of all developing maps of $g_{\text {st }}$ can be identified with the group PSU(2). Up to taking the reciprocal, any two developing maps of $g_{\text {st }}$, fixing 0 and $\infty$, respectively, differ by a multiple complex constant with modulus 1 . Up to sign, the logarithmic differentials of all the developing maps, leaving the set $\{0, \infty\}$ invariant, coincide with the abelian differential $\Theta:=d(\log w)=d w / w$, which has two simple poles of 0 and $\infty$. The residues of $\Theta$ at 0 and $\infty$ equal 1 and -1 , respectively. The algebraic dual $X:=w \partial / \partial w$ of $\Theta$ is a meromorphic vector field with two simple zeroes of 0 and $\infty$. The index of $X$ equals 1 at both 0 and $\infty . \Phi(w)=4|w|^{2} /\left(1+|w|^{2}\right)$ is a smooth Morse function on $\overline{\mathbb{C}}$, whose complex gradient field $\Phi^{\cdot}, w \partial / \partial w$ equals $X$. Moreover, $\Phi$ has only two critical points, which are the minimal point 0 and the maximal point $\infty$. Consider a multiplicative developing map $f$ of a reducible metric $g$ on $\Sigma$. Up to sign, the character 1-form $\omega=d f / f=d(\log f)$ equals the pullback $f^{*} \Theta$ of $\Theta$ by $f$. Denote by $Y:=\left(f(z) / f^{\prime}(z)\right)(\partial / \partial z)$ the algebraic dual vector field of $\omega$, which is a meromorphic vector field on $\Sigma$. Then $Y$ equals the complex gradient field $\Psi^{\cdot}, z \partial / \partial z$ of the smooth function $\Psi(z)=4|f(z)|^{2} /\left(1+|f(z)|^{2}\right)$ on $\Sigma^{*}$, which can be continuously extended to $\Sigma$ (see Lemma 4.3). 
Using the above notations, we state more precisely the properties of the character 1 -form of a reducible metric.

Theorem 1.4. Let $g$ be a reducible metric representing the divisor

$$
D=\sum_{j=1}^{n}\left(\alpha_{j}-1\right) P_{j} \quad \text { with } 1 \neq \alpha_{j}>0 .
$$

Let $f$ be a developing map of $g$, and let $f$ be multiplicative if $g$ is nontrivial such that the character 1 -form $\omega=d f / f$ of $g$ equals $f^{*} \Theta$. Let $Y$ be the algebraic dual vector field of $\omega$, and $\Psi(z)=4|f(z)|^{2} /\left(1+|f(z)|^{2}\right)$. Then the following statements hold:

(1) The set of zeroes of the meromorphic vector field $Y$ coincides with the extremal point set of the function $\Psi$. Each zero of $Y$ is simple, and $Y$ vanishes at each point $p_{j}$ where $\alpha_{j}>0$ is a noninteger. The set of poles of $Y$ coincides with the saddle point set of $\Psi$. Each pole of $Y$ is some conical singularity $p_{j}$ of the reducible metric $g$, where $\alpha_{j}$ is an integer greater than 1 and the order of the pole $p_{j}$ of $Y$ equals $\alpha_{j}-1$.

(2) Let $p_{1}, \ldots, p_{J}$ be the saddle points of $\Psi$, let $p_{J+1}, \ldots, p_{n}$ be the singular extremal points of $\Psi$, and let $e_{1}, \ldots, e_{S}$ be the smooth extremal points of $\Psi$ on $\Sigma^{*}$. Then the canonical divisor of the character 1-form $\omega$ has the form

$$
(\omega)=\sum_{j=1}^{J}\left(\alpha_{j}-1\right) P_{j}-\sum_{k=J+1}^{n} P_{k}-\sum_{\ell=1}^{S} E_{\ell} .
$$

In particular, each pole of $\omega$ is simple; that is, $\omega$ is an abelian differential of the third kind. The residue of $\omega$ at the pole $e_{\ell}$ equals 1 or -1 , where $e_{\ell}$ is a minimal or maximal point of $\Psi$; the residue of $\omega$ at the pole $p_{k}$ equals $\alpha_{j}$ or $-\alpha_{j}$, where $p_{k}$ is a minimal or maximal point of $\Psi$. Moreover the real part of $\omega$ is exact on $\Sigma^{\prime}:=\Sigma \backslash\left\{p_{J+1}, \ldots, p_{n}, e_{1}, \ldots, e_{S}\right\}:$

$$
2 \Re \omega=d\left(\log |f|^{2}\right) .
$$

(3) The developing map $f$ extends over $\Sigma^{\prime} \cup \Sigma^{*}$ and has the expression

$$
f(z)=C \exp \left(\int^{z} \omega\right)
$$

for some nonzero complex constant $C$. In particular, the local monodromy of $f$ around each $p_{j}(1 \leq j \leq J)$ is trivial, and the limit $\lim _{p \rightarrow p_{j}} f(p)$ exists and belongs to $\mathbb{C} \backslash\{0\}$. If we continue analytically a function element $\mathfrak{f}$ of $f$ along a simple and sufficiently small loop winding around $p_{k}(J+1 \leq k \leq n)$ counterclockwise, then we obtain $\mathfrak{f} \exp \left(2 \pi \sqrt{-1} \alpha_{k}\right)$. The limit $\lim _{p \rightarrow p_{k}}|f(p)|$ exists, and equals 0 or $+\infty$, provided $p_{k}$ is a minimal or maximal point of $\Psi(z)$. This is also the case for $e_{\ell}$. 
Using abelian differentials of the third kind with the above properties, we can construct new examples of conformal metrics with constant curvature one and with finitely many conical singularities.

Theorem 1.5. Let $\omega$ be an abelian differential of the third kind having poles on a compact Riemann surface $\Sigma$, whose residues are all nonzero real numbers and whose real part is exact outside the set of poles of $\omega$. Then there exists a unique 1-parameter family $\left\{g_{\lambda}: \lambda \in(0,+\infty)\right\}$ of reducible metrics on $\Sigma$ such that $\omega$ is one of the character 1 -forms of each metric $g_{\lambda}:=f_{\lambda}^{\star} g_{\text {st }}$, where

$$
f_{\lambda}(z)=\lambda \cdot \exp \left(\int^{z} \omega\right)
$$

is a multivalued locally univalent meromorphic function on $\Sigma \backslash\{$ poles of $\omega\}$ with monodromy in $\mathrm{U}(1)$. Suppose that the canonical divisor of $\omega$ has the form

$$
(\omega)=\sum_{j=1}^{J}\left(\alpha_{j}-1\right) P_{j}-\sum_{k=J+1}^{N} Q_{k},
$$

where the $\alpha_{j}$ are integers $>1$. Then the divisor $D$ represented by $g_{\lambda}$ has the form

$$
D=\sum_{j=1}^{J}\left(\alpha_{j}-1\right) P_{j}+\sum_{k=J+1}^{N}\left(\left|\operatorname{Res}_{Q_{k}}(\omega)\right|-1\right) Q_{k} .
$$

Moreover, the $g_{\lambda}$ are trivial reducible metrics if and only if the integral of $\omega$ on each loop in $\Sigma \backslash\{$ poles of $\omega\}$ is $2 \pi \sqrt{-1}$ times an integer. In particular, each residue of $\omega$ is an integer.

Remark 1.6. Each reducible metric does not satisfy Troyanov's condition (1) (see Corollary 4.5). Therefore we obtain a class of new examples of conformal metrics of constant curvature one with finitely many singularities, since there exist plenty of abelian differentials of the third kind satisfying the condition in Theorem 1.5 (see [Springer 1957, Corollary 8-3]).

Troyanov's condition (1) for the corresponding irreducible metrics depends only on the values of angles. Example 4.7 shows that the existence of reducible metrics does not depend only on angles but also on the position of singularities.

Remark 1.7. Umehara and Yamada [2000] called a nontrivial reducible metric $\mathscr{H}^{1}$-reducible, and a trivial reducible metric $\mathscr{H}^{3}$-reducible.

Remark 1.8. Besides the previous reference, our motivation for defining character 1 -form comes from [Chen and Wu 2011; Chen et al. 2013], where the authors use character 1-form to completely classify HCMU metrics of nonconstant curvature on compact Riemann surfaces. We will say more about this in the ending of Section 4. 
Theorem 1.9. A conformal metric of constant curvature one, representing an effective $\mathbb{Z}$-divisor $D$ on the 2-sphere is a trivial reducible metric. That is, it is the pullback under some rational function $f$ on the 2-sphere of the standard metric $g_{\text {st }}$ on the Riemann sphere $\overline{\mathbb{C}}$.

Remark 1.10. The case where $D$ is an effective $\mathbb{Z}$-divisor supported at two or three points was proved in [Troyanov 1989; Furuta and Hattori 1998; Umehara and Yamada 2000; Eremenko 2004]. However Theorem 1.9 does not hold in general for compact Riemann surfaces of nonzero genus (see Example 4.6).

Since the rational function $f$ in Theorem 1.9 has ramified divisor $D$, the theorem reduces the problem of characterizing conformal metrics of constant curvature one and representing an effective $\mathbb{Z}$-divisor $D$ on the two-sphere to the following classical one: Which kind of divisors $D$ can be a ramification divisor of some rational function, and how many equivalent classes of rational functions have the prescribed ramification divisor? Here we say that two rational functions are equivalent if one of them is given by the postcomposition of the other with a Möbius transformation.

When the points in the support of the ramified divisor lie in a general position, L. Goldberg [1991] solved a special case of the latter problem, where each ramified order equals one; I. Scherbak [2002] gave a complete answer for the general case. [Eremenko and Gabrielov 2002; Eremenko et al. 2006] proved that there exists a real rational function in each equivalence class if each point in the support of $D$ is real.

Remark 1.11. Since we only consider conformal metrics with finite area in this manuscript, the singularities of "zero angle" would not show up (see [Bryant 1988, Proposition 4]).

We explain the organization of this paper. In Section 2, we shall first make a detailed exposition on developing map in Lemmas 2.1 and 2.2. We compute, in Lemma 3.1 of Section 3, the Schwarzian of a developing map of a conformal metric with constant curvature one representing a divisor $D$. In Lemma 3.2 we show that the converse of Lemma 3.1 also holds. Then Theorem 1.9 follows from these two lemmas. In Section 4 we prove Theorems 1.4 and 1.5 as applications of Lemma 3.2. These two theorems are applied to give some examples of irreducible and reducible metrics in Corollary 4.5 and Examples 4.6 and 4.7. In Section 5, we give an alternative proof of a theorem of Troyanov [1989], as an application of Theorems 1.9 and 1.4. Moreover we discuss the nonuniqueness of reducible metrics representing a given divisor $D$. Last, we propose some questions about both irreducible and reducible metrics. 


\section{Existence of developing maps and their monodromy}

Lemma 2.1. Let $g$ be a conformal metric on a compact Riemann surface $\Sigma$ of constant curvature one, representing the divisor $D=\sum_{j=1}^{n}\left(\alpha_{j}-1\right) P_{j}$ with $\alpha_{j}>0$. Then there exists a multivalued locally univalent holomorphic map $f$ from $\Sigma^{*}:=$ $\Sigma \backslash\left\{p_{1}, \ldots, p_{n}\right\}$ to the Riemann sphere $\overline{\mathbb{C}}$ such that the monodromy of $f$ belongs to $\mathrm{PSU}(2)$ and

$$
g=f^{*} g_{\mathrm{st}},
$$

where $g_{\mathrm{st}}=4|d w|^{2} /\left(1+|w|^{2}\right)^{2}$ is the standard metric over $\overline{\mathbb{C}}$.

Proof. Denote by $d(\cdot, \cdot)$ the distance on $\Sigma$ induced by the metric $g$. Choose an arbitrary point $p$ in $\Sigma^{*}$ and fix it. Take a positive number $r=r_{p}$ sufficiently small such that $d\left(p,\left\{p_{1}, \ldots, p_{n}\right\}\right)>r$ and there exists a geodesic polar coordinate chart in the open metric ball $B(p, r)=\{q \in \Sigma \mid d(p, q)<r\} \subset \Sigma^{*}$. Choose a positively oriented orthonormal basis $\left\{e^{1}, e^{2}\right\}=\left\{e_{p}^{1}, e_{p}^{2}\right\}$ of the tangent space $T_{p} \Sigma$. Choose an arbitrary point $\mathfrak{p} \in \overline{\mathbb{C}}$ and fix it. Since the Gauss curvature of $(B(p, r), g)$ is constant and equals one, by a theorem of Riemann [Petersen 2006, p. 136], there exists an open metric ball $\mathfrak{B}(\mathfrak{p}, r)$ in the Riemann sphere $\left(\overline{\mathbb{C}}, g_{\mathrm{st}}\right)$ and an orientationpreserving isometry $\mathfrak{f}_{p}$ from $(B(p, r), g)$ onto $\left(\mathfrak{B}(\mathfrak{p}, r), g_{\text {st }}\right)$. Let $\mathfrak{e}_{\mathfrak{p}}^{1}:=\mathfrak{f}_{*}\left(e^{1}\right)$ and $\mathfrak{e}_{\mathfrak{p}}^{2}:=\mathfrak{f}_{*}\left(e^{2}\right)$, which also form a positively oriented orthonormal basis of $T_{\mathfrak{p}} \overline{\mathbb{C}}$. Then $\mathfrak{f}_{p}$ is a conformal map from $B(p, r)$ to $\mathfrak{B}(\mathfrak{p}, r)$.

Take an arbitrary point $q$ in $\Sigma^{*}$ and a curve $L:[0,1] \rightarrow \Sigma^{*}$ joining $p$ to $q$. Then there exists some

$$
0<\delta<\min \left(d\left(\gamma([0,1]),\left\{p_{1}, \ldots, p_{n}\right\}\right), r_{p}\right)
$$

such that there exists a geodesic polar coordinate chart in the open metric ball $B(a, \delta)$ for each point $a$ on the curve $L$. If we properly divide the interval $0 \leq t \leq 1$ into $n$ subintervals for sufficiently large $n, 0=\gamma_{0}<\gamma_{1}<\cdots<\gamma_{n}=1$, then the curve $L$ splits into $n$ subarcs $L_{1}, L_{2}, \ldots, L_{n}$ with $L_{g}(g=1, \ldots, n)$ joining $L\left(\gamma_{g-1}\right)=: c_{g-1}$ to $L\left(\gamma_{g}\right)=: c_{g}$. Moreover, if we denote by $B_{0}, B_{1}, \ldots, B_{n}$ the open metric balls with centers $a=c_{0}, c_{1}, \ldots, c_{n}$ and with radius $\delta$, then the closed arcs $L_{g}$ lie completely in $B_{g-1}$ for $g=1, \ldots, n$. Let $f_{0}$ be the restriction to $B_{0}$ of the conformal map $\mathfrak{f}_{p}: B\left(p, r_{p}\right) \rightarrow \mathfrak{B}\left(\mathfrak{p}, r_{p}\right)$. Then $f_{0}$ is an isometry (conformal map) from $B_{0}$ onto $\mathfrak{B}_{0}:=\mathfrak{B}(\mathfrak{p}, \delta)$. Choose a positively oriented orthonormal basis $\left\{e_{c_{1}}^{1}, e_{c_{2}}^{2}\right\}$ of $T_{c_{1}} \Sigma$. Since $c_{1} \in B_{0}$, we let $\mathfrak{c}_{1}:=f_{0}\left(c_{1}\right) \in \mathfrak{B}_{0}, \mathfrak{e}_{c_{1}}^{1}:=\left(f_{0}\right)_{*}\left(e_{c_{1}}^{1}\right)$ and $\mathfrak{e}_{c_{1}}^{2}:=\left(f_{0}\right)_{*}\left(e_{c_{1}}^{2}\right)$. Then there exists a unique isometry $f_{1}: B_{1} \rightarrow \mathfrak{B}_{1}:=\mathfrak{B}\left(\mathfrak{c}_{1}, \delta\right)$ such that $f_{1}\left(c_{1}\right)=\mathfrak{c}_{1}$ and $f_{1}$ maps $\left\{e_{c_{1}}^{1}, e_{c_{1}}^{2}\right\}$ to $\left\{\mathfrak{e}_{\mathfrak{c}_{1}}^{1}, \mathfrak{e}_{\mathfrak{c}_{1}}^{2}\right\}$. Then $f_{1}=f_{0}$ on $B_{0} \cap B_{1}$. Since $c_{2} \in L_{2} \subset B_{1}$, $f_{1}$ is an analytic continuation of $f_{0}$ from point $c_{0}$ to $c_{2}$ along the $\operatorname{arc} L_{0} \cup L_{1}$. In this way, we obtain $f_{0}, \ldots, f_{n}$, which are recursively defined on $B_{0}, \ldots, B_{n}$ and give an analytic continuation of $\mathfrak{f}_{p}$ from $p$ to $q$ along the curve $L$. Using the same 
argument as [Siegel 1969, pp. 13-15], we can show that this analytic continuation is independent of the choice of division points on $L$. Moreover, if $L^{*}$ is another curve in $\Sigma^{*}$ joining $a$ to $b$ that is homotopic to $L$, then the result of doing analytic continuation of $\mathfrak{f}_{p}$ along $L^{*}$ is the same as along $L$. Summing up, we obtain a multivalued locally isometric (univalent conformal) map $f$ from $\left(\Sigma^{*}, g\right)$ to $\left(\overline{\mathbb{C}}, g_{\text {st }}\right)$.

At last, we prove that all the monodromy of $f$ belongs to PSU(2). Suppose $L:[0,1] \rightarrow \Sigma^{*}$ is a closed curve with $L(0)=L(1)=p$. We use the notation in the previous paragraph. Recall that $f_{0}$ maps $p=c_{0}$ to $\mathfrak{p},\left(f_{0}\right)_{*}$ maps $\left\{e^{1}, e^{2}\right\}$ to $\left\{\mathfrak{e}_{\mathfrak{p}}^{1}, \mathfrak{e}_{\mathfrak{p}}^{2}\right\}, f_{n}$ maps $p=c_{n}$ to $\mathfrak{c}_{n}$ and $\left(f_{n}\right)_{*}$ maps $\left\{e^{1}, e^{2}\right\}$ to $\left\{\mathfrak{e}_{\mathfrak{c}_{n}}^{1}, \mathfrak{e}_{\mathfrak{c}_{n}}^{2}\right\}$. Then there exists a unique isometry $\mathfrak{L} \in \operatorname{PSU}(2)$ of $\left(\overline{\mathbb{C}}, g_{\text {st }}\right)$ such that $\mathfrak{L}(\mathfrak{p})=\mathfrak{c}_{n}$ and $\mathfrak{L}_{*}$ maps $\left\{\mathfrak{e}_{\mathfrak{p}}^{1}, \mathfrak{e}_{\mathfrak{p}}^{2}\right\}$ to $\left\{\mathfrak{e}_{\mathfrak{c}_{n}}^{1}, \mathfrak{e}_{\mathfrak{c}_{n}}^{2}\right\}$. Therefore $f_{n}=\mathfrak{L} \circ f_{0}$.

Lemma 2.2. Any two developing maps $f_{1}, f_{2}$ of the metric $g$ are related by a fractional linear transformation $\mathfrak{L} \in \mathrm{PSU}(2)$, i.e., $f_{2}=\mathfrak{L} \circ f_{1}$. In particular, any two developing maps of $g$ have mutually conjugate monodromy in PSU(2). Then we call this conjugate class the monodromy of the metric $g$. The space of developing maps of the metric $g$ has a one-to-one correspondence with the quotient group of $\mathrm{PSU}(2)$ by the monodromy group of a developing map of $g$.

Proof. Take a point $p \in \Sigma^{*}$ and a positively oriented orthonormal basis $\left\{e^{1}, e^{2}\right\}$ of $T_{p} \Sigma^{*}$. Let $\mathfrak{f}_{j}$ be a function element of $f_{j}$ near $p$ for $j=1,2$. Denote $\mathfrak{p}_{j}:=\mathfrak{f}_{j}(p)$ and $\mathfrak{e}_{\mathfrak{p}_{j}}^{k}:=\left(\mathfrak{f}_{j}\right)_{*}\left(e^{k}\right)$ for $j, k=1,2$. Then there exists a unique $\mathfrak{L} \in \operatorname{PSU}(2)$ such that $\mathfrak{L}\left(\mathfrak{p}_{1}\right)=\mathfrak{p}_{2}$, and $\mathfrak{L}_{*}$ maps $\left\{\mathfrak{e}_{\mathfrak{p}_{1}}^{1}, \mathfrak{e}_{\mathfrak{p}_{1}}^{2}\right\}$ to $\left\{\mathfrak{e}_{\mathfrak{p}_{2}}^{1}, \mathfrak{e}_{\mathfrak{p}_{2}}^{2}\right\}$. Then we obtain the equality $\mathfrak{f}_{2}=\mathfrak{L} \circ \mathfrak{f}_{1}$ near $p$, which implies $f_{2}=\mathfrak{L} \circ f_{1}$. It follows from direct computation that the monodromy of $f_{1}$ and $f_{2}$ are mutually conjugate.

Given a developing map $f$ and a fractional linear transformation $\mathfrak{L} \in \operatorname{PSU}(2)$, we can see $\mathfrak{L} \circ f=f$ if and only if there exists a point $p \in \Sigma^{*}$ and a functional element $\mathfrak{f}_{p}$ near $p$ such that $\mathfrak{L} \circ \mathfrak{f}_{p}$ is another function element of $f$ near $p$. That is, $\mathfrak{L}$ belongs to the image of the monodromy representation of $\pi_{1}\left(\Sigma^{*}, p\right)$ with respect to $f$. Therefore PSU(2) acts in this way transitively on the set of all developing maps with isotropy group isomorphic to the monodromy group.

Remark 2.3. The developing maps also exist for flat or hyperbolic conformal metrics with finitely many conical or cusp singularities, and analogues of Lemmas 2.2 and 3.2 hold.

\section{The Schwarzian of a developing map}

Lemma 3.1. Let $g$ be a conformal metric of constant curvature one on a compact Riemann surface $\Sigma$, and suppose g represents a divisor $D=\sum_{j=1}^{n}\left(\alpha_{j}-1\right) P_{j}$, where $\alpha_{j}>0$ for all $j$. Suppose that $f: \Sigma^{*}=\Sigma \backslash\left\{p_{1}, \ldots, p_{n}\right\} \rightarrow \overline{\mathbb{C}}$ is a developing 
map of $g$. Then the Schwarzian $\{f, z\}$ of $f$ equals

$$
\{f, z\}=\frac{1-\alpha_{j}^{2}}{2 z^{2}}+\frac{d_{j}}{z}+\psi_{j}(z)
$$

in a neighborhood $U_{j}$ of $p_{j}$ with complex coordinate $z$ and $z\left(p_{j}\right)=0$, where the $d_{j}$ are constants and the $\psi_{j}$ are holomorphic functions in $U_{j}$, depending on the complex coordinate $z$.

Proof. If we rewrite the metric $g=4\left|f^{\prime}(z)\right|^{2}|d z|^{2} /\left(1+|f(z)|^{2}\right)^{2}$ as $g=e^{2 u}|d z|^{2}$, then we find $u=\log \left|f^{\prime}(z)\right|+\log 2-\log \left(1+|f|^{2}\right)$. The lemma on page 300 of [Troyanov 1989] tells us that

$$
\eta(z)=2\left(\frac{\partial^{2} u}{\partial z^{2}}-\left(\frac{\partial u}{\partial z}\right)^{2}\right) d z^{2}
$$

defines a projective connection compatible with the divisor $D$. The interested reader could find in the same reference the definition of the projective connection, which we will not use in this paper. The compatibility of the projective connection $\eta$ with the divisor $D$ on page 300 of [Troyanov 1989] means that

$$
\eta(z)=\left(\frac{1-\alpha_{j}^{2}}{2 z^{2}}+\frac{d_{j}}{z}+\phi_{j}(z)\right) d z^{2}, \quad \phi_{j} \text { holomorphic, }
$$

where $z$ is the complex coordinate near $p_{j}$. Since the developing map $f$ is a projective multivalued function on $\Sigma^{*}$, its Schwarzian $\{f, z\}$ with respect to the complex coordinate $z$ near $p_{j}$ is a single-valued function of $z$. At last, we find

$$
\begin{aligned}
2\left(\frac{\partial^{2} u}{\partial z^{2}}-\left(\frac{\partial u}{\partial z}\right)^{2}\right)= & 2 \frac{\partial}{\partial z}\left(\frac{f^{\prime \prime}(z)}{2 f^{\prime}(z)}-\frac{f^{\prime}(z) \bar{f}}{1+|f|^{2}}\right)-2\left(\frac{f^{\prime \prime}(z)}{2 f^{\prime}(z)}-\frac{f^{\prime}(z) \bar{f}}{1+|f|^{2}}\right)^{2} \\
= & \left(\frac{f^{\prime \prime \prime}(z)}{f^{\prime}(z)}-\left(\frac{f^{\prime \prime}(z)}{f^{\prime}(z)}\right)^{2}-\frac{2 f^{\prime \prime}(z) \bar{f}(z)}{1+|f|^{2}}+2\left(\frac{f^{\prime}(z) \bar{f}}{1+|f|^{2}}\right)^{2}\right) \\
& \quad-\left(\frac{1}{2}\left(\frac{f^{\prime \prime}(z)}{f^{\prime}(z)}\right)^{2}-\frac{2 f^{\prime \prime}(z) \bar{f}(z)}{1+|f|^{2}}+2\left(\frac{f^{\prime}(z) \bar{f}}{1+|f|^{2}}\right)^{2}\right) \\
= & \frac{f^{\prime \prime \prime}(z)}{f^{\prime}(z)}-\frac{3}{2}\left(\frac{f^{\prime \prime}(z)}{f^{\prime}(z)}\right)^{2}=\{f, z\} .
\end{aligned}
$$

A multivalued locally univalent meromorphic function $h$ on $\Sigma^{*}$ is said to be projective if any two function elements $\mathfrak{h}_{1}, \mathfrak{h}_{2}$ of $h$ near a point $p \in \Sigma^{*}$ are related by a fractional linear transformation $L \in \operatorname{PGL}(2, \mathbb{C})$, i.e., $\mathfrak{h}_{1}=L \circ \mathfrak{h}_{2}$.

Lemma 3.2. Let $f: \Sigma^{*} \rightarrow \overline{\mathbb{C}}$ be a projective multivalued locally univalent meromorphic function, and suppose the monodromy of $f$ belongs to a maximal compact subgroup of $\mathrm{PGL}(2, \mathbb{C})$. If $f$ is compatible with the divisor $D=\sum_{j}\left(\alpha_{j}-1\right) P_{j}$, then 
there exists a neighborhood $U_{j}$ of $p_{j}$ with complex coordinate $z$ and $L_{j} \in \operatorname{PGL}(2, \mathbb{C})$ such that $z\left(p_{j}\right)=0$ and $g_{j}=L_{j} \circ f$ has the form $g_{j}(z)=z^{\alpha_{j}}$, where $0<\alpha_{j} \neq 1$ and $c_{j}=\left(1-\alpha_{j}^{2}\right) / 2$. Moreover there exists $\mathfrak{L} \in \operatorname{PGL}(2, \mathbb{C})$ such that the pullback $(\mathfrak{L} \circ f)^{*} g_{\text {st }}$ of the standard metric $g_{\text {st }}$ by $\mathfrak{L} \circ f$ is a conformal metric of constant curvature one which represents the divisor $D=\sum_{j}\left(\alpha_{j}-1\right) P_{j}$. In particular, if the monodromy of $f$ belongs to PSU(2), then the fractional linear transformation $\mathfrak{L}$ turns out to be the identity map.

Proof. Recall the well-known fact that every maximal compact group of PGL $(2, \mathbb{C})$ is conjugate to the subgroup PSU(2). There exists a fractional linear transformation $\mathfrak{L}$ such that the monodromy of $\mathfrak{L} \circ f$ belongs to PSU(2). Hence we may assume that this is the case for $f$ without loss of generality.

We first show the first statement of Lemma 3.2: that there exists a neighborhood $U_{j}$ of $p_{j}$ with complex coordinate $z$ and some $L_{j} \in \operatorname{PGL}(2, \mathbb{C})$ such that $g_{j}=L_{j} \circ f$ has the form $z^{\alpha_{j}}$. Since $f$ is compatible with $D$, we could choose a neighborhood $U_{j}$ of $p_{j}$ and a complex coordinate $x$ on $U_{j}$ such that $x\left(p_{j}\right)=0$ and

$$
\{f, x\}=\frac{c_{j}}{x^{2}}+\frac{d_{j}}{x}+\phi_{j}(x),
$$

where $\phi_{j}(x)$ is holomorphic in $U_{j}$ and $c_{j}:=\left(1-\alpha_{j}^{2}\right) / 2$. By [Yoshida 1987, Proposition, p. 39], in the neighborhood $U_{j}$ there are two linearly independent solutions $u_{0}$ and $u_{1}$ of the equation

$$
\frac{d^{2} u}{d x^{2}}+\frac{1}{2}\left(\frac{c_{j}}{x^{2}}+\frac{d_{j}}{x}+\phi_{j}(x)\right) u=0,
$$

with single-valued coefficient such that $f(x)=u_{1}(x) / u_{0}(x)$. Actually we have $u_{0}=(d f / d x)^{-1 / 2}$ and $u_{1}=f(x) u_{0}$. Moreover, if $f$ changes projectively, i.e.,

$$
f \mapsto \frac{a f+b}{c f+d} \quad \text { with } a d-b c=1,
$$

then $u_{0}$ and $u_{1}$ change linearly, i.e.,

$$
\left(\begin{array}{l}
u_{0} \\
u_{1}
\end{array}\right) \mapsto\left(\begin{array}{ll}
d & c \\
b & a
\end{array}\right)\left(\begin{array}{l}
u_{0} \\
u_{1}
\end{array}\right)
$$

and vice versa.

Define an operator $L_{j}:=x^{2}\left(d^{2} / d x^{2}\right)+q_{j}(x)$ with $q_{j}(x)=\left(c_{j}+d_{j} x+x^{2} \phi_{j}(x)\right) / 2$. Then both $u_{0}$ and $u_{1}$ are solutions of the equation $L_{j} u=0$. Since the monodromy of $f$ belongs to PSU(2), the cyclic group generated by the local monodromy of the equation $L_{j} u=0$ around $x=0$ is contained in a maximal compact subgroup of $\operatorname{PGL}(2, \mathbb{C})$ conjugate to $\operatorname{PSU}(2)$. Note that the equation $L_{j} u=0$ has a regular singularity at 0 . We could apply the Frobenius method (see [Yoshida 1987, §2.5]) 
to solve it. Note that the indicial equation

$$
f(s)=s(s-1)+\frac{1-\alpha_{j}^{2}}{4}=0
$$

of the differential equation $L_{j} u=0$ at $x=0$ has roots $s_{0}=\left(1-\alpha_{j}\right) / 2$ and $s_{1}=\left(1+\alpha_{j}\right) / 2$, and $s_{1}-s_{0}=\alpha_{j}>0$. Let $\sum_{k=0}^{\infty} b_{k} x^{k}$ be the power series expansion of $q_{j}(x)$, where $b_{0}=c_{j} / 2$. Let $s$ be a parameter. Then $u(s, x)=x^{s} \sum_{k=0}^{\infty} c_{k}(s) x^{k}$, with $c_{0}(s) \equiv 1$, is an solution of $L_{j} u=0$ if and only if the equation

$$
f(s+n) c_{n}+R_{n}=0
$$

holds for all $n=0,1,2, \ldots$, where

$$
R_{0}=0, \quad \text { and, for } n>0, \quad R_{n}=R_{n}\left(c_{1}, \ldots, c_{n-1}, s\right)=\sum_{i=0}^{n-1} c_{i} b_{n-i} .
$$

Note that the equation $\left(\sharp_{0}\right)$ is exactly the indicial equation (2). Since $f\left(s_{1}+n\right) \neq 0$ for all $n \geq 1$, we find that $u\left(s_{1}, x\right)$ is a solution of the equation.

Case 1. Suppose that $s_{1}-s_{0}=\alpha_{j}$ is not an integer. Then, by the same reasoning, $u\left(s_{0}, x\right)$ is another solution, which is linearly independent of $u\left(s_{1}, x\right)$. Summing up, we have

$$
u\left(s_{0}, x\right)=x^{s_{0}}\left(1+\psi_{0}\right) \quad \text { and } \quad u\left(s_{1}, x\right)=x^{s_{1}}\left(1+\psi_{1}\right),
$$

where both $\psi_{0}$ and $\psi_{1}$ are holomorphic functions vanishing at 0 . Here we take a smaller neighborhood of 0 than $U_{j}$ to assure the convergence of the power series defining $\psi_{k}$ if necessary. Since both $u_{0}(x)$ and $u_{1}(x)$ are linear combinations of $u\left(s_{0}, x\right)$ and $u\left(s_{1}, x\right), f(x)=u_{1}(x) / u_{0}(x)$ equals some fractional linear transform of $u\left(s_{1}, x\right) / u\left(s_{0}, x\right)$. For simplicity of notation, we may assume $f(x)=u\left(s_{1}, x\right) / u\left(s_{0}, x\right)$ equals $x^{\alpha_{j}}$ times a holomorphic function $\varphi_{j}(x)$ with $\varphi_{j}(0)=1$. Therefore we could choose another complex coordinate $z=z(x)$ of $U_{j}$ under which $f=f(z)=z^{\alpha_{j}}$.

Case 2. Suppose that $m:=s_{1}-s_{0}=\alpha_{j}$ is an integer $\geq 2$.

Subcase 2.1. If $R_{m}=0$, we can solve the equation $\left(\sharp_{n}\right)$ for $s=s_{0}$ for all $n \geq 1$ by choosing $c_{m}$ arbitrarily and obtain another solution $u\left(s_{0}, x\right)$ linearly independent of $u\left(s_{1}, x\right)$. An argument similar to that in Case 1 completes the proof.

Subcase 2.2. Suppose $R_{m} \neq 0$. Define

$$
u^{*}=x^{s_{0}} \sum_{k=0}^{\infty} c_{k}\left(s_{0}\right) x^{k}
$$

where $c_{0}=1$, the $c_{j}(1 \leq j<m)$ are determined by $\left(\sharp_{j}\right)$, while $c_{m}$ is arbitrarily fixed, and the $c_{j}(j>m)$ are determined also by $\left(\sharp_{j}\right)$. Then the linear combination 
of $u^{*}$ and $\left.(\partial / \partial s) u(s, x)\right|_{s=s_{1}}$

$$
U_{0}(x):=f^{\prime}\left(s_{1}\right) u^{*}-\left.R_{m} \frac{\partial}{\partial s} u(s, x)\right|_{s=s_{1}}
$$

is a solution. It should be mentioned that since $f$ and $R_{n}$ are holomorphic with respect to $s$, this is also the case for the $c_{n}$. Then we correct a typo in [Yoshida 1987, p. 23] and find the two linearly independent solutions, given by

$$
\left(\begin{array}{c}
U_{0}(x) \\
u\left(s_{1}, x\right)
\end{array}\right)=\left(\begin{array}{cc}
x^{s_{0}} & x^{s_{1}} \log x \\
0 & x^{s_{1}}
\end{array}\right)\left(\begin{array}{c}
f^{\prime}\left(s_{1}\right) \sum_{k=0}^{\infty} c_{k}\left(s_{0}\right) x^{k}-R_{m} x^{m} \sum_{k=0}^{\infty} c_{k}^{\prime}\left(s_{1}\right) x^{k} \\
\sum_{k=0}^{\infty} c_{k}\left(s_{1}\right) x^{k}
\end{array}\right) .
$$

Then the local monodromy of the equation $L_{j} u=0$ at $x=0$ is the conjugacy class in $\operatorname{PGL}(2, \mathbb{C})$ of the matrix

$$
M=\left(\begin{array}{cc}
1 & 2 \pi \sqrt{-1} \\
0 & 1
\end{array}\right)
$$

However, the cyclic group generated by $M$ is a free abelian group, which has no limit point under the usual topology of $\operatorname{PGL}(2, \mathbb{C})$, contradicting the fact that the monodromy of the equation $L_{j} u=0$ is contained in a compact group of PGL $(2, \mathbb{C})$. That is, we rule out Subcase 2.2.

Summing up, we prove the statement where $\alpha_{j}$ is an integer $\geq 2$. Moreover we can also see that in this case the local monodromy at $p_{j}$ is trivial; that is, $p_{j}$ is an apparent singularity of the equation $L_{j} u=0$ and the multivalued function $f$.

Since $f$ is locally univalent on $\Sigma^{*}$ and has monodromy belonging to $\operatorname{PSU}(2)$, $f^{*} g_{\text {st }}$ is a well-defined smooth Riemannian metric on $\Sigma^{*}$ with constant curvature one. The first statement proved just now implies that this metric has conical singularities at $p_{j}$ with angles $2 \pi \alpha_{j}$.

Remark 3.3. Lemma 3.2 has some overlap with [Bryant 1988, Proposition 4], in the sense that both say the same thing near each singularity.

We sum up the above two lemmas:

Theorem 3.4. There exists a conformal metric of constant curvature one representing a divisor $D$ on a compact Riemann surface $\Sigma$ if and only if there is a projective multivalued meromorphic function on $\Sigma^{*}=\Sigma \backslash$ Supp D compatible with the divisor $D$ and having monodromy in PSU(2).

Proof of Theorem 1.4. Let $f$ be a developing map of the metric $g$ representing an effective $\mathbb{Z}$-divisor $\sum_{j} n_{j} P_{j}$ on the sphere. By Lemma 3.1, $f$ has regular singularity of weight $\left(1-n_{j}^{2}\right) / 2$ at $p_{j}$. By Lemma 2.1, the monodromy of $f$ belongs to PSU(2). By Lemma 3.2, there exists $\mathfrak{L}_{j} \in \operatorname{PGL}(2, \mathbb{C})$ and a complex coordinate $z$ near $p$ such that $\mathfrak{L}_{j} \circ f$ has the form $f(z)=z^{n_{j}+1}$ near $p_{j}$, which implies the local monodromy of $f$ at $p_{j}$ is trivial. Since the sphere is simply connected, the monodromy of $f$ is 
trivial, that is, $f$ is a single-valued meromorphic function outside $\left\{p_{j}\right\}$. Moreover, $f$ can be extended meromorphically onto the whole sphere; that is, $f$ is a rational function on the sphere.

At a point $p \in \Sigma$ near which $f=f(z)$ is univalent holomorphic, we find that the Schwarzian $\{f, z\}$ is holomorphic, and vice versa (see [Yoshida 1987, Remark, p. 44]). Actually we can prove a more general result.

Lemma 3.5. Let $U$ be an open disk containing 0 in the complex plane $\mathbb{C}$ with coordinate $w$ and $f$ a projective multivalued meromorphic function on $U \backslash\{0\}$ with regular singularity of weight zero at 0 . That is, $\{f, w\}$ equals $d / w$ plus a holomorphic function $\phi(w)$, where both the constant $d$ and $\phi(w)$ depend on the coordinate $w$. Assume that the subgroup of $\operatorname{PGL}(2, \mathbb{C})$ generated by the local monodromy of $f$ at 0 is precompact in $\operatorname{PGL}(2, \mathbb{C})$. Then there exists $\mathfrak{L} \in \operatorname{PGL}(2, \mathbb{C})$ and another complex coordinate $z$ of $U$ such that $\mathfrak{L} \circ f(z)=z$ and $z(0)=0$.

Proof. Use the same argument as Case 2 of the proof of Lemma 3.2. Also note that the indicial equation here has two roots, 0 and 1.

This lemma has the following geometric consequence.

Proposition 3.6. The conic singularity with angle $2 \pi$ of a conformal metric with constant curvature one is actually a smooth point of the metric.

\section{Proof of Theorems 1.5 and 1.9}

Lemma 4.1. (1) A subgroup $G$ of PSU(2) can be diagonalized if and only if $G$ has a fixed point on $\overline{\mathbb{C}}$. Such a group is contained in some maximal torus $\mathbb{\mathbb { T }}$ of PSU(2). In particular, $G$ is abelian.

(2) There exists an abelian subgroup of PSU(2) which has no fixed point on $\overline{\mathbb{C}}$.

Proof. (1) Consider the natural unitary representation $\rho$ of $\mathrm{SU}(2)$ on $V \cong \mathbb{C}^{2}$ endowed with the natural Hermitian inner product $\langle\cdot, \cdot\rangle$. For each subgroup $H$ of $\mathrm{SU}(2), \rho$ restricts to a faithful unitary representation $\rho_{H}$ of $H$ on $V$. Let $\widetilde{G} \subset \mathrm{SU}(2)$ be the lifting of $G \subset \mathrm{PSU}(2)$. We say that $G$ can be diagonalized if the representation $\left(\rho_{\widetilde{G}}, V\right)$ can be decomposed into the direct sum of two one-dimensional subspaces:

$$
V=\mathbb{C} e_{1} \oplus \mathbb{C} e_{2} \quad \text { and } \quad\left\langle e_{k}, e_{\ell}\right\rangle=\delta_{k \ell} .
$$

Then, up to conjugacy, $\widetilde{G}$ can be viewed as a subgroup of the standard maximal torus

$$
\mathrm{U}(1)=\left\{\operatorname{diag}\left(e^{\sqrt{-1} \theta}, e^{-\sqrt{-1} \theta}\right) \mid \theta \in \mathbb{R}\right\}
$$

of $\mathrm{SU}(2)$. Hence $G=\widetilde{G} /\left\{ \pm I_{2}\right\}$ is abelian.

Looking at the Riemann sphere $\overline{\mathbb{C}}$ as the complex one-dimensional projective space $\mathbb{P}(V)$ with the natural projection $\pi: V \backslash\{0\} \rightarrow \mathbb{P}(V)$, we can see that both 
$\pi\left(e_{1}\right)$ and $\pi\left(e_{2}\right)$ are two distinct fixed points of the $G$-action on $\mathbb{P}(V)$ if $G$ can be diagonalized.

Suppose that $G$ has a fixed point $\pi\left(e_{1}\right)$ on $\mathbb{P}(V)$ with $e_{1} \in V$ and $\left\langle e_{1}, e_{1}\right\rangle=1$. Then $e_{1}$ is a common eigenvector of all the elements in $\widetilde{G}$. Since $\rho_{\widetilde{G}}$ is a unitary representation on $V$, it can be decomposed into $V=\mathbb{C} e_{1} \oplus \mathbb{C} e_{2}$, where $e_{2}$ is a unit vector orthogonal to $e_{1}$. That is, $G$ can be diagonalized.

(2) The abelian subgroup $D_{2}$ of PSU(2) generated by

$$
z \mapsto-z \quad \text { and } \quad z \mapsto \frac{1}{z}
$$

has no fixed point on $\overline{\mathbb{C}}$.

Lemma 4.2. A trivial reducible metric $g$ representing a divisor $D$ on a compact Riemann surface $\Sigma$ is the pullback $f^{*} g_{\mathrm{st}}$ of $g_{\mathrm{st}}$ by some rational function $f$ on $\Sigma$.

Proof. Let $f: \Sigma^{*} \rightarrow \overline{\mathbb{C}}$ be a developing map of the metric $g$. Since the monodromy of $f$ is trivial, so is the local monodromy of $f$ around each point in the support of $D$. By Lemma 3.2 the divisor $D$ must be an effective $\mathbb{Z}$-divisor. Using Lemma 3.2 again, we find that the holomorphic map $f: \Sigma^{*} \rightarrow \overline{\mathbb{C}}$ has a holomorphic extension to $\Sigma$.

Lemma 4.3. Suppose that $g$ is a reducible metric on $\Sigma$ and $f$ a multiplicative developing map of $g$. Then the holomorphic 1 -form $d(\log f)$ on $\Sigma^{*}$ can be extended to be an abelian differential of the third kind on $\Sigma$. The function $\Psi=4|f(z)|^{2} /\left(1+|f(z)|^{2}\right)$ on $\Sigma^{*}$ can be extended continuously to $\Sigma$.

Proof. The proof is contained in the following proof of Theorem 1.4.

Proof of Theorem 1.4. (1-2) We show that if a point $p \in \Sigma^{*}$ is a zero of $Y(z)=$ $\left(f(z) / f^{\prime}(z)\right)(\partial / \partial z)$, then $p$ is simple. We choose a function element $\mathfrak{f}$ near $p$. Since the monodromy of $f$ belongs to $\mathrm{U}(1), Y=\left(\mathfrak{f}(z) / \mathfrak{f}^{\prime}(z)\right) \partial / \partial z$, which is independent of the choice of the function element $\mathfrak{f}$ and the complex coordinate $z$. Since $\mathfrak{f}$ is a univalent meromorphic function near $p$, there exist $\mathfrak{L} \in \operatorname{PGL}(2, \mathbb{C})$ and a complex coordinate $z$ near $p$ with $z(p)=0$ such that $\mathfrak{L} \circ f=z$. Then $\mathfrak{f}=(a z+b) /(c z+d)$ with $a d-b c=1$ near $p$, and $Y=(a z+b)(c z+d) \partial / \partial z$. It is clear that $p$ cannot be a pole of $Y$. Since $Y=0$ at $z(p)=0, b d=0$.

Case 1. Since $a d-b c=1$ and $b d=0$, we assume $b=0, d \neq 0$ in this case. Then $a d=1$ and $\mathfrak{f}(z)=a z /(c z+d)$. Then $Y=a z(c z+d) \partial / \partial z$ has a simple zero at $z(p)=0$. Hence $\omega=d z /(a z(c z+d))$ has residue 1 at $p$. Since $f(0)=0, p$ is a minimal point of $\Psi$ and $\Psi(p)=0$.

Case 2. Similarly, when $d=0$ and $b \neq 0, Y$ also has simple zero at $p, \omega$ has residue $-1, \lim _{q \rightarrow p}|f(q)|=+\infty, \lim _{q \rightarrow p} \Psi(q)=4$, and $p$ is a maximal point of $\Psi$. 
We show that each point $q \in\left\{p_{1}, \ldots, p_{n}\right\}$ must be a simple zero of $Y$, provided the conical angle of the metric $g$ at $q$ equals $2 \pi s>0$ and $s$ is a noninteger. By Lemmas 3.1 and 3.2, we can choose a function element $\mathfrak{f}$ near $q$ and a complex coordinate $z$ near $q$ such that $\mathfrak{f}=\left(a z^{s}+b\right) /\left(c z^{s}+d\right)$ with $a d-b c=1$. On the other hand, since the monodromy of $f$ belongs to $\mathrm{U}(1)$, so does the local monodromy of $\mathfrak{f}$. Then there exists $\theta \in \mathbb{R}$ such that

$$
e^{2 \pi \sqrt{-1} \theta} \mathfrak{f}=e^{2 \pi \sqrt{-1} \theta} \frac{a z^{s}+b}{c z^{s}+d}=\frac{a e^{2 \pi \sqrt{-1} s} z^{s}+b}{c e^{2 \pi \sqrt{-1} s} z^{s}+d} .
$$

This is equivalent to the following equalities holding:

$$
\begin{aligned}
& a c e^{2 \pi \sqrt{-1} s}\left(1-e^{2 \pi \sqrt{-1} \theta}\right)=0, \\
& \left(a d e^{2 \pi \sqrt{-1} s}+b c\right)-e^{2 \pi \sqrt{-1} \theta}\left(b c e^{2 \pi \sqrt{-1} s}+a d\right)=0, \\
& b d\left(1-e^{2 \pi \sqrt{-1} \theta}\right)=0 .
\end{aligned}
$$

Solving the equation, we find that either $c=b=0$ or $a=d=0$, that is, $\mathfrak{f}(z)$ equals $\mu z^{s}(\mu \neq 0)$ or $\lambda z^{-s}(\lambda \neq 0)$. Hence $Y= \pm s z(\partial / \partial z)$ has a simple zero at $z(q)=0$. Since $\mathfrak{f}(0)$ equals 0 or $\infty, \Psi$ is continuous at $q$, which is a minimal or maximal point of $\Psi$ achieving value 0 or 4 if and only if $\omega$ has residue $s>0$ or $-s<0$ at $p$.

Let $p$ be a singular point of the metric $g$ with conical angle $2 \pi$ times an integer $n>1$. Then

$$
\mathfrak{f}(z)=\frac{a z^{n}+b}{c z^{n}+d} \quad \text { and } \quad Y=\frac{\left(a z^{n}+b\right)\left(c z^{n}+d\right)}{n z^{n-1}} \frac{\partial}{\partial z} .
$$

Case A. Assume $b d \neq 0$. Then $p$ is a pole of $Y$ with order $n-1$ and a zero of $\omega$ with order $n-1$, and $\lim _{z \rightarrow p} f(z)=f(0)=b / d \in \mathbb{C} \backslash\{0\}$. Moreover $\Psi$ is continuous at $p$, which is a saddle point of $\Psi$.

Case B. Assume $b d=0$. Then it is easy to check that $p$ is a simple zero of $Y$. If $b=0$ (resp. $b \neq 0$ ), then $\lim _{q \rightarrow p}|f(p)|$ equals 0 (resp. $+\infty$ ), where $\omega$ has residue $n$ (resp. $-n$ ). $\Psi$ is continuous at $p$, where it achieves the minimal value 0 or the maximal value 4 .

(3) The local monodromy property of $f$ follows from Lemmas 3.1 and 3.2.

Lemma 4.4. (1) Let $g$ be a reducible metric on $\Sigma$. Then each character 1-form $\omega$ of $g$ has at least two poles.

(2) Besides the assumption of (1), assume that $\omega$ has no zero and has only two poles. Then $\Sigma$ is the Riemann sphere $\overline{\mathbb{C}}$ and $g$ has two singularities with the same angle, say $\alpha>0$. Moreover, if the two singularities are assumed to be 0 and $\infty$, then $\omega=\alpha(d z / z)$ up to sign. 
Proof. (1) Let $f$ be the multiplicative developing map such that $\omega=d(\log f)$. Since $\Psi=4|f|^{2} /\left(1+|f|^{2}\right)$ is a nonconstant continuous function on $\Sigma$, it must achieve its minimum and maximum. Either a minimal point or a maximal one of $\Psi$ is a pole of $\omega$ by Theorem 1.4.

(2) $\Sigma=\overline{\mathbb{C}}$ follows from $\operatorname{deg}(\omega)=-2$. By the residue theorem, the two residues of $\omega$ have different signs at the two poles, say 0 and $\infty$. It follows from Theorem 1.4 that $g$ has exactly two singularities 0 and $\infty$ with the same angle, say $\alpha$. Assume that $\omega$ has residues $\alpha$ and $-\alpha$ at 0 and $\infty$, respectively. Then $\omega=\alpha(d z / z)$.

Proof of Theorem 1.5. We divide the proof into the following two cases:

Case 1. Assume that the integral of $\omega$ at some loop in $\Sigma^{\prime}:=\Sigma \backslash\{$ poles of $\omega$ \} does not belong to the set $2 \pi \sqrt{-1} \mathbb{Z}$. Since $\Re \omega$ is exact on $\Sigma^{\prime}$, solving the equation

$$
\omega=d(\log f)
$$

on $\Sigma^{\prime}$, up to a complex multiple with modulus one, we obtain a 1-parameter family of multivalued locally univalent meromorphic functions

$$
f_{\lambda}(z)=\lambda \cdot \exp \left(\int^{z} \omega\right), \quad \lambda \in(0,+\infty)
$$

Moreover, $f_{\lambda}$ has nontrivial monodromy belonging to $\mathrm{U}(1)$ and $f_{\lambda}^{\star} g_{\mathrm{st}}$ is a nontrivial reducible metric with character 1 -form $\omega$. Conversely, if $g$ is a reducible metric such that $\omega$ is one of its character 1-forms, then there exists a developing map $\tilde{f}$ of $g$ such that $\omega=d(\log \tilde{f})$. Since $\tilde{f}$ has nontrivial monodromy in $\mathrm{U}(1), g$ is nontrivial. Solving the equation $\omega=d \tilde{f} / \tilde{f}$, up to a complex multiple with modulus one, we find that $\tilde{f}$ equals $f_{\lambda}$ for some $\lambda>0$. Therefore such a reducible metric $g$ is unique. By the argument in the proof of Theorem 1.4, we find the divisor $D$ represented by $g$ equals

$$
\sum_{j=1}^{J}\left(\alpha_{j}-1\right) P_{j}+\sum_{k=J+1}^{N}\left(\left|\operatorname{Res}_{Q_{k}}(\omega)\right|-1\right) Q_{k} .
$$

Case 2. Assume that the monodromy given by $\omega$ is trivial; that is, the integral of $\omega$ at each loop in $\Sigma^{\prime}:=\Sigma \backslash$ poles of $\omega$ \} belongs to the set $2 \pi \sqrt{-1} \mathbb{Z}$. The pullback $f^{*} g_{\text {st }}$ with $f(z)=\exp \left(\int^{z} \omega\right)$ is a trivial reducible metric such that $f$ is one of its developing map and a rational function on $\Sigma$ and $\omega$ is one of its character 1-forms. Conversely, if $g$ is a reducible metric with $\omega$ one of its character 1-forms, then $g=\tilde{f}^{*} g_{\text {st }}$ with $\tilde{f}(z)=\lambda \exp \left(\int^{z} \omega\right)$ for some $\lambda>0$. Moreover, $\tilde{f}$ is a rational function uniquely determined by $\omega$ and $\lambda$. Therefore, such a reducible metric $g$ lies in the 1-parameter family $\left\{f_{\lambda}^{*} g_{\text {st }}: \lambda \in(0,+\infty)\right\}$. By a similar argument to the proof 
of Theorem 1.4, we can show that the effective divisor represented by $g$ equals

$$
\sum_{j=1}^{J}\left(\alpha_{j}-1\right) P_{j}+\sum_{k=J+1}^{N}\left(\left|\operatorname{Res}_{Q_{k}}(\omega)\right|-1\right) Q_{k} .
$$

Corollary 4.5. Under the notation of Theorem 1.5, we have

$$
\chi(\Sigma)+\operatorname{deg} D \geq \min \left(2,2 \min _{j} \alpha_{j}\right) .
$$

In particular, the divisor D does not satisfy Troyanov's condition (1). In other words, if $D$ satisfies condition (1), then each conformal metric which has constant curvature one and represents $D$ is irreducible.

Proof. The character 1-form $\omega$ has at least two poles since the continuous function $\Psi=4|f|^{2} /\left(1+|f|^{2}\right)$ in Theorem 1.4 has at least a minimal point and a maximal one. Then, using the equality

$$
-\chi(\Sigma)=\operatorname{deg}(\omega)=\sum_{j=1}^{J}\left(\alpha_{j}-1\right)-(N-J),
$$

we have

$$
\begin{aligned}
\chi(\Sigma)+\operatorname{deg} D & =\chi(\Sigma)+\sum_{j=1}^{J}\left(\alpha_{j}-1\right)+\sum_{k=J+1}^{N}\left(\left|\operatorname{Res}_{Q_{k}}(\omega)\right|-1\right) \\
& =\sum_{k=J+1}^{N}\left|\operatorname{Res}_{Q_{k}}(\omega)\right| \geq \min \left(2,2 \min _{j} \alpha_{j}\right) .
\end{aligned}
$$

Example 4.6. Consider a conformal metric $g$ on the two-sphere with constant curvature one and finitely many conical singularities $p_{1}, \ldots, p_{n}$. Let the angle at $p_{j}$ be $2 \pi \alpha_{j}$. If $n \geq 3$ and each $\alpha_{j}$ is a noninteger, then $g$ is irreducible. Otherwise, by Theorem 1.4, the character 1 -form of $g$ would have at least three poles and have no zeroes, a contradiction. In particular, we consider the conformal metric $g$ of constant curvature one with three angles $\pi, \pi, \pi$ at 0,1 and $\infty$ on the twosphere. Then the developing maps of $g$ coincide with those projective solutions with monodromy in PSU(2) of the Gauss hypergeometric equation

$$
z(1-z) \frac{d^{2} u}{d z^{2}}+(c-(a+b+1) z) \frac{d u}{d z}-a b u=0,
$$

where

$$
|1-c|=|c-a-b|=|a-b|=\frac{1}{2}
$$

(see [Yoshida 1987, Section 5.3]). Then the monodromy group of a developing map of $g$ is conjugate to $D_{2}$ in Lemma 4.1 and thus abelian, but $g$ is irreducible. 
It follows from [Troyanov 1991, Theorem 4] that on a torus there exists a conformal metric $g$ with constant curvature one and a conical singularity $p$ with angle $2 \pi \alpha$, where $1<\alpha<3$. Then Corollary 4.5 tell us that $g$ is not reducible. The existence of such a irreducible metric having one angle $4 \pi$ implies that Theorem 1.9 does not hold on a torus.

Example 4.7. We at first consider an elementary example of reducible metrics on the Riemann sphere $\overline{\mathbb{C}}$. Let $a, b$ be two positive numbers. Consider the 1 -form

$$
\omega=\left(\frac{a}{z}+\frac{b}{z-1}\right) d z,
$$

which has residue $-a-b$ at $\infty$. Note that $a /(a+b)$ is the zero of $\omega$. Hence $\omega$ satisfies the condition in Theorem 1.5 and thus gives a reducible metric on the two-sphere with angles $2 \pi a, 2 \pi b, 2 \pi(a+b)$ and $4 \pi$ at $0,1, \infty$ and $a /(a+b)$, respectively.

On the other hand, suppose that $g$ is a reducible metric on the two-sphere having angles $2 \pi a, 2 \pi b, 2 \pi(a+b)$ and $4 \pi$ at $0,1, \infty$ and $\lambda \in \mathbb{C} \backslash\{0,1\}$, respectively, where $a, b, a+b$ are not integers. Then $\lambda=a /(a+b)$. Actually, letting $\omega$ be a character 1 -form of $g$, we can see from Theorem 1.4 that $0,1, \infty$ are simple poles of $\omega$, and $\lambda$ is the zero of $\omega$. By the residue theorem, we may assume that

$$
\begin{aligned}
& \operatorname{Res}_{0}(\omega)=a, \\
& \operatorname{Res}_{1}(\omega)=b, \\
\operatorname{Res}_{\infty}(\omega)= & -a-b,
\end{aligned}
$$

which implies that

$$
\omega=\left(\frac{a}{z}+\frac{b}{z-1}\right) d z \quad \text { and } \quad \lambda=\frac{a}{a+b} .
$$

We then give an explicit construction of a general reducible metric $g$ on the Riemann sphere $\overline{\mathbb{C}}$. Suppose that $z_{1}, \ldots, z_{k}, k \geq 2$, are all the poles of $\omega$ lying in $\mathbb{C}$. Then

$$
\omega=\left(\sum_{j=1}^{k} \frac{a_{j}}{z-z_{j}}\right) d z,
$$

where $a_{j} \in \mathbb{R} \backslash\{0\}$ is the residue of $\omega$ at $z_{j}$ for each $j$. Hence the zero set of $\omega$ is determined by the poles of $\omega$ and their residues. In other words, the set $\left\{\left(z_{j}, a_{j}\right): j=1,2, \ldots, k\right\}$ determines the positions of the singularities of $g$ corresponding to the saddle points of the function $\Psi$ and the values of their conical angles. Solving the ordinary differential equation $d \log f=\omega$, we find that there 
exists some $\lambda>0$ such that the multivalued function

$$
f_{\lambda}(z)=\lambda \cdot \prod_{j=1}^{k}\left(z-z_{j}\right)^{a_{j}}
$$

is a developing map of the metric $g$. This implies that the existence of reducible metrics does not only depend on angles, but also on the position of singularities.

Remark 4.8. The monodromy of a developing map is irreducible for a hyperbolic conformal metric with finitely many conical or cusp singularities. Moreover it is also the case for a flat conformal metric with finitely many conical singularities unless the metric is a smooth one on a torus. We leave the proof to interested readers.

We conclude this section by saying something more about the relationship between reducible metrics and HCMU metrics. As a generalization with singularities on compact Riemann surfaces of Calabi's extremal Kähler metric on compact complex manifolds [Calabi 1982; 1985], X. Chen [2000] first introduced the concepts of the HCMU metric and the extremal metric with singularities, and proved some fundamental results. In particular, a conformal metric $\tilde{g}$ on a compact Riemann surface with singularities is called $H C M U$ if and only if it has finite area and finite Calabi energy, and the complex gradient $K^{\cdot}, z \partial / \partial z$ of the Gauss curvature $K=K_{\tilde{g}}$ is a holomorphic vector field outside the singularities. Wang and Zhu [2000] and Lin and Zhu [2002] obtained some interesting results and generalized some results of X. Chen. Recently [Chen and Wu 2011; Chen et al. 2013] completely classified the nonconstant curvature HCMU metrics with conical or cusp singularities, by using the character 1-form

$$
\tilde{\omega}=\frac{d z}{K_{\tilde{g}}^{\cdot z}}
$$

of an HCMU metric $\tilde{g}$. The properties that $\tilde{\omega}$ is an abelian differential of the third kind with real residues and its real part is exact outside the set of simple poles play a crucial rule in the classification. The following observation puts both HCMU metrics and metrics of constant curvature in the same philosophical frame.

Observation 4.9. Given a nonconstant curvature HCMU metric $\tilde{g}$ with singularities on a compact Riemann surface $\Sigma$, there exists a multivalued locally univalent meromorphic function $\tilde{f}$ on $\Sigma^{*}$ having monodromy in the abelian group

$$
\{\exp (\sqrt{-1} \theta) \mid \theta \in \mathbb{R}\},
$$

and a football HCMU metric $g_{\mathrm{fb}}$ (see [Chen 2000; Chen et al. 2005]) over $\overline{\mathbb{C}}$ such that $\tilde{g}=\tilde{f}^{*} g_{f b}$. Moreover the character 1-form of $\tilde{g}$ coincides with the logarithmic differential $d(\log f)$ of $f$, up to a constant.

This is not used in this paper and its proof will be left elsewhere. 


\section{Discussion}

As an application of Theorems 1.9 and 1.4, we shall show that if $g$ is a conformal metric on the sphere $\overline{\mathbb{C}}$ of constant curvature one, representing the divisor $D=$ $(\alpha-1) P+(\beta-1) Q$, where $\alpha, \beta>0$, then $\alpha=\beta$.

Proof. Case 1. We assume that at least one of $\alpha$ and $\beta$ is not an integer. Suppose that this is the case for $\alpha$. Since the punctured sphere $\overline{\mathbb{C}} \backslash\{p, q\}$ has fundamental group isomorphic to $\mathbb{Z}$, the metric $g$ is a reducible metric. Let $f$ be one of its developing maps. By Lemmas 3.1 and 3.2, the local monodromy of $f$ at $p$ is nontrivial. Hence $g$ is nontrivial. We may assume that $f$ is multiplicative so that $\omega=d f / f$ is the character 1-form of $g$. Theorem 1.4 tells us that $p$ is a simple pole of $\omega$ with residue $\pm \alpha$, and $q$ is either a simple pole or a zero point of $\omega$. If $q$ is a simple pole too, then the residue equals $\pm \beta$. Since the canonical divisor $(\omega)$ has degree -2 , by Theorem 1.4, we find that $\omega$ has exactly two poles of $p, q$, which implies $\alpha=\beta$. It is impossible that $q$ is a zero point of $\omega$; otherwise $\omega$ must have at least two simple poles in $\overline{\mathbb{C}} \backslash\{p, q\}$, each of which has residue \pm 1 by Theorem 1.4. This contradicts the fact that $\alpha \notin \mathbb{Z}$.

Case 2. Suppose that both $\alpha$ and $\beta$ are integers $\geq 2$. By Theorem 1.9, each developing map $f$ of $g$ is a rational function on $\overline{\mathbb{C}}$. Since the ramification divisor $R_{f}$ of $f$ equals $(\alpha-1) P+(\beta-1) Q, f$ has degree $d=(\alpha+\beta) / 2$ by the RiemannHurwitz theorem. Suppose $\alpha \neq \beta$, say $\alpha>\beta$. Then $f$ has expression $z \mapsto z^{\alpha}$ near $p$, which implies $f$ has degree $\geq \alpha>d$. Contradiction!

We observe that the reducible metrics representing a given divisor are not unique in general:

Proposition 5.1. Suppose that there exists a reducible metric $g$ representing the divisor $D$ on the two-sphere. Denote by $\mathbb{M}(D)$ the space of conformal metrics of constant curvature one representing $D$, by $\mathbb{A}(D)$ that of reducible metrics representing $D$.

(1) If $D$ is supported at two points $p_{1}$ and $p_{2}, \mathbb{M}(D)=\mathbb{A}(D)$, and $g$ is unique if and only if the two angles are equal and do not belong to $2 \pi \mathbb{Z}_{>1}$. If the two angles are equal and belong to $2 \pi \mathbb{Z}_{>1}$, then $\mathbb{A}(D)$ is connected and has dimension 1 .

(2) If $D$ is supported at three points and $\mathbb{A}(D) \neq \varnothing$, then $\mathbb{M}(D)=\mathbb{A}(D)$ is connected. Moreover if $g$ is trivial, $\operatorname{dim} \mathbb{A}(D)=3 ;$ otherwise $\operatorname{dim} \mathbb{A}(D)=1$.

(3) Suppose that $D$ is supported at more than three points and $\mathbb{A}(D) \neq \varnothing$. Then, if $g$ is trivial, $\operatorname{dim} \mathbb{A}(D)=3$; otherwise $\operatorname{dim} \mathbb{A}(D) \geq 1$.

Proof. The first statement was proved by Troyanov [1989, Theorem I, p. 298]. The second was shown in [Umehara and Yamada 2000, Corollary 2.3]. 
Suppose that $D$ is supported at more than three points. Following [Umehara and Yamada 2000, (2.5)], we define

$$
I_{g}:=\left\{g_{a}=(a \star f)^{*} g \mid a \in \operatorname{PSL}(2, \mathbb{C}), a \cdot \operatorname{Im} \rho_{f} \cdot a^{-1} \subset \operatorname{PSU}(2)\right\},
$$

where $a \star f$ denotes the Möbius transformation of $f$ by $a$ and

$$
\rho_{f}: \pi_{1}\left(\Sigma^{*}\right) \rightarrow \operatorname{PSU}(2)
$$

denotes the monodromy representation of the developing map $f$ of the metric $g$. Each metric $g_{a}$ in $I_{g}$ has a developing map $a \star f$, which has the same Schwarzian as $f$ and monodromy conjugate to that of $f$. Hence $I_{g}$ is contained in $\mathbb{A}(D)$. Then it follows from [Umehara and Yamada 2000, Lemma B, p. 92] that if $g$ is trivial, $\operatorname{dim} \mathbb{A}(D) \geq 3$; otherwise $\operatorname{dim} \mathbb{A}(D) \geq 1$.

We consider the moduli of trivial reducible metrics representing an effective $\mathbb{Z}$-divisor $D$, which can be reduced to the space of rational functions with the same ramification divisor $D$. We say that two rational functions are equivalent if one of them is given by the postcomposition of the other with a Möbius transformation. It follows from [Umehara and Yamada 2000, Lemma B, p. 92] that the trivial reducible metrics having developing maps of the same type form a moduli of the three-dimensional hyperbolic space $\mathscr{H}^{3}$. The beautiful theorem in [Scherbak 2002] says that there is a least upper bound given by the Schubert calculus for the number of equivalent classes of all the rational functions with ramification divisor $D$, which can be achieved by a generic choice of the support of $D$. Hence we obtain the corresponding information for the number of connected components of $\mathbb{A}(D)$.

It is time to propose some questions interesting to us.

Question 5.2. Does there exist a divisor on some compact Riemann surface which is represented by both an irreducible metric and a reducible one? It does not happen for the divisors satisfying the Troyanov condition (1) by Corollary 4.5. It also does not happen on the two-sphere under either of the following two conditions:

(1) The support of $D$ consists of three points or less (see Proposition 5.1).

(2) Each $\alpha_{j}$ is a noninteger for $j=1,2, \ldots, n$, with $n \geq 3$ (see Example 4.6).

Question 5.3. Suppose $\mathbb{A}(D)$ is nonempty for a divisor $D$ on a compact Riemann surface. Study the moduli space $\mathbb{A}(D)$ of reducible metrics representing a divisor $D$ on a compact Riemann surface, such as its dimension and the number of its components. We know the answer on the two-sphere in the case that $D$ has support at two or three points or $D$ is an effective $\mathbb{Z}$-divisor in Proposition 5.1.

Question 5.4. Suppose that there exists an irreducible metric $g$ representing $D=$ $\sum_{j}\left(\alpha_{j}-1\right) P_{j}$. Is $g$ the unique metric of constant curvature one representing $D$ ? Luo and Tian [1992] showed this is the case on the two-sphere if each $\alpha_{j}$ lies in 
$(0,1)$. Moreover, Umehara and Yamada [2000] gave a positive answer if $D$ is a divisor supported at three points on the two-sphere.

Question 5.5. Suppose that there exists an irreducible metric $g$ representing $D=$ $\sum_{j}\left(\alpha_{j}-1\right) P_{j}$. Does there exist an irreducible metric representing any divisor $D^{\prime}$ sufficiently near $D$ ? On the two-sphere, if each $\alpha_{j}$ lies in $(0,1)$, then the necessary and sufficient condition is a topologically open one for the existence of a irreducible metric representing $D$ given by Troyanov [1991] and Luo and Tian [1992]. On the two-sphere, if $D$ has support at three points, so is the necessary and sufficient condition given by Umehara and Yamada [2000]. S. K. Donaldson [2012] proved an openness theorem for Kähler Einstein metrics on a Fano manifold with conical singularity along the anticanonical divisor.

\section{Acknowledgments}

$\mathrm{Xu}$ would like to express his deep gratitude to Professor Xiuxiong Chen for his constant moral support and lots of invaluable conversations. He thanks Professor Masaaki Umehara so much for many valuable comments and discussions through emails. He is also very grateful to Professor Ryoichi Kobayashi, Dr. Zhi Chen and Dr. Jinxing Xu for their stimulating conversations. In particular, the penetrating questions from Dr. Jinxing Xu gave him the impetus to fix a gap in the old version of the manuscript.

\section{Note added in proof}

After this paper was accepted, the authors learned that Daniele Bartolucci, Francesca De Marchis and Andrea Malchiodi [Bartolucci et al. 2011] proved a general existence result for the problem of prescribing the Gaussian curvature on surfaces of positive genus with conical singularities in supercritical regimes. Two years later, Bartolucci and Malchiodi [2013] removed the assumption on the genus. As a consequence, their results imply a new general existence theorem for conformal metrics with constant curvature one and finitely many conical singularities which do not satisfy the Troyanov condition (1). The authors observed that these metrics are irreducible. Moreover, Bartolucci, De Marchis and Malchiodi [Bartolucci et al. 2011] proved the existence of multiple solutions on surfaces of genus bigger than one, which implies that the answer to Question 5.4 is negative.

\section{References}

[Bartolucci and Malchiodi 2013] D. Bartolucci and A. Malchiodi, "An improved geometric inequality via vanishing moments, with applications to singular Liouville equations", Comm. Math. Phys. 322:2 (2013), 415-452. MR 3077921 Zbl 1276.58005 
[Bartolucci et al. 2011] D. Bartolucci, F. De Marchis, and A. Malchiodi, "Supercritical conformal metrics on surfaces with conical singularities", Int. Math. Res. Not. 2011:24 (2011), 5625-5643. MR 2863376 Zbl 1254.30066

[Bryant 1988] R. L. Bryant, "Surfaces of mean curvature one in hyperbolic space", pp. 321-347 in Théorie des variétés minimales et applications (Palaiseau, 1983-1984), Astérisque 154-155, Société Mathématique de France, Paris, 1988. MR 955072 Zbl 0635.53047

[Calabi 1982] E. Calabi, “Extremal Kähler metrics”, pp. 259-290 in Seminar on Differential Geometry, edited by S.-T. Yau, Ann. of Math. Stud. 102, Princeton University Press, 1982. MR 83i:53088 Zbl 0487.53057

[Calabi 1985] E. Calabi, "Extremal Kähler metrics, II", pp. 95-114 in Differential geometry and complex analysis, edited by I. Chavel and H. M. Farkas, Springer, Berlin, 1985. MR 86h:53067 Zbl 0574.58006

[Chen 2000] X. Chen, "Obstruction to the existence of metric whose curvature has umbilical Hessian in a K-surface”, Comm. Anal. Geom. 8:2 (2000), 267-299. MR 2001k:53060 Zbl 0971.53029

[Chen and Wu 2011] Q. Chen and Y. Wu, "Character 1-form and the existence of an HCMU metric", Math. Ann. 351:2 (2011), 327-345. MR 2012g:58020 Zbl 1227.53076

[Chen et al. 2005] Q. Chen, X. Chen, and Y. Wu, "The structure of HCMU metric in a $K$-surface", Int. Math. Res. Not. 2005:16 (2005), 941-958. MR 2006f:53046 Zbl 1090.53036

[Chen et al. 2013] Q. Chen, Y. Wu, and B. Xu, "HCMU metrics with cusp and conic singularities", preprint, 2013. arXiv 1302.6655

[Donaldson 2012] S. K. Donaldson, "Kähler metrics with cone singularities along a divisor", pp. 49-79 in Essays in mathematics and its applications, edited by P. M. Pardalos and T. M. Rassias, Springer, Heidelberg, 2012. MR 2975584

[Eremenko 2004] A. Eremenko, "Metrics of positive curvature with conic singularities on the sphere", Proc. Amer. Math. Soc. 132:11 (2004), 3349-3355. MR 2005h:53054 Zbl 1053.53025

[Eremenko and Gabrielov 2002] A. Eremenko and A. Gabrielov, "Rational functions with real critical points and the B. and M. Shapiro conjecture in real enumerative geometry", Ann. of Math. (2) 155:1 (2002), 105-129. MR 2003c:58028 Zbl 0997.14015

[Eremenko et al. 2006] A. Eremenko, A. Gabrielov, M. Shapiro, and A. Vainshtein, "Rational functions and real Schubert calculus", Proc. Amer. Math. Soc. 134:4 (2006), 949-957. MR 2007d:14103 Zbl 1110.14052

[Fujimori et al. 2011] S. Fujimori, Y. Kawakami, M. Kokubu, W. Rossman, M. Umehara, and K. Yamada, "CMC-1 trinoids in hyperbolic 3-space and metrics of constant curvature one with conical singularities on the 2-sphere”, Proc. Japan Acad. Ser. A Math. Sci. 87:8 (2011), 144-149. MR 2012k:53013 Zbl 1242.53070

[Furuta and Hattori 1998] M. Furuta and Y. Hattori, "2-dimensional singular spherical space forms", manuscript, 1998.

[Goldberg 1991] L. R. Goldberg, "Catalan numbers and branched coverings by the Riemann sphere", Adv. Math. 85:2 (1991), 129-144. MR 92b:14014 Zbl 0732.14013

[Lin and Zhu 2002] C. S. Lin and X. Zhu, "Explicit construction of extremal Hermitian metrics with finite conical singularities on $S^{2}$,, Comm. Anal. Geom. 10:1 (2002), 177-216. MR 2003c:58010 Zbl 1021.58008

[Luo and Tian 1992] F. Luo and G. Tian, "Liouville equation and spherical convex polytopes", Proc. Amer. Math. Soc. 116:4 (1992), 1119-1129. MR 93b:53034 Zbl 0806.53012

[McOwen 1988] R. C. McOwen, "Point singularities and conformal metrics on Riemann surfaces", Proc. Amer. Math. Soc. 103:1 (1988), 222-224. MR 89m:30089 Zbl 0657.30033 
[Petersen 2006] P. Petersen, Riemannian geometry, 2nd ed., Graduate Texts in Mathematics 171, Springer, New York, 2006. MR 2007a:53001 Zbl 1220.53002

[Scherbak 2002] I. Scherbak, "Rational functions with prescribed critical points", Geom. Funct. Anal. 12:6 (2002), 1365-1380. MR 2004c:14101 Zbl 1092.14065

[Siegel 1969] C. L. Siegel, Topics in complex function theory, I: Elliptic functions and uniformization theory, Interscience Tracts in Pure and Applied Mathematics 25, Wiley, New York, 1969. MR 41 \#1977 Zbl 0184.11201

[Springer 1957] G. Springer, Introduction to Riemann surfaces, Addison-Wesley, Reading, MA, 1957. MR 19,1169g Zbl 0078.06602

[Troyanov 1989] M. Troyanov, "Metrics of constant curvature on a sphere with two conical singularities”, pp. 296-306 in Differential geometry (Peñíscola, 1988), edited by F. J. Carreras et al., Lecture Notes in Math. 1410, Springer, Berlin, 1989. MR 90m:53057 Zbl 0697.53037

[Troyanov 1991] M. Troyanov, "Prescribing curvature on compact surfaces with conical singularities", Trans. Amer. Math. Soc. 324:2 (1991), 793-821. MR 91h:53059 Zbl 0724.53023

[Umehara and Yamada 2000] M. Umehara and K. Yamada, "Metrics of constant curvature 1 with three conical singularities on the 2-sphere", Illinois J. Math. 44:1 (2000), 72-94. MR 2001f:53072 Zbl 0958.30029

[Wang and Zhu 2000] G. Wang and X. Zhu, "Extremal Hermitian metrics on Riemann surfaces with singularities", Duke Math. J. 104:2 (2000), 181-210. MR 2001i:58017 Zbl 0980.58009

[Yoshida 1987] M. Yoshida, Fuchsian differential equations: with special emphasis on the GaussSchwarz theory, Aspects of Mathematics 11, Vieweg, Braunschweig, 1987. MR 90f:32025 Zbl 0618.35001

Received July 20, 2013. Revised December 18, 2013.

\section{QING CHEN}

Wu Wen-Tsun Key Laboratory of Math, UStC, Chinese Academy of Sciences

SCHOOL OF MATHEMATICAL SCIENCES

UNIVERSITY OF SCIENCE AND TECHNOLOGY OF CHINA

HEFEI, 230026

CHINA

qchen@ustc.edu.cn

WEI WANG

DEPARTMENT OF MATHEMATICS

UNIVERSITY OF CALIFORNIA, IRVINE

IRVINE, CA 92697-3875

UNITED STATES

wangw10@math.uci.edu

\section{YINGYI WU}

SCHOOL of Mathematical SCIENCES

UNIVERSITY OF CHINESE ACADEMY OF SCIENCES

BEIJING, 100049

CHINA

wuyy@ucas.ac.cn 
BIN XU

Wu Wen-Tsun Key Laboratory of Math, UStC, Chinese Academy of Sciences SCHOOL OF MATHEMATICAL SCIENCES

UNIVERSITY OF SCIENCE AND TECHNOLOGY OF CHINA

HEFEI, 230026

CHINA

bxu@ustc.edu.cn 


\title{
PACIFIC JOURNAL OF MATHEMATICS
}

\author{
msp.org/pjm
}

Founded in 1951 by E. F. Beckenbach (1906-1982) and F. Wolf (1904-1989)

\section{EDITORS}

Don Blasius (Managing Editor)

Department of Mathematics

University of California

Los Angeles, CA 90095-1555

blasius@math.ucla.edu

\author{
Paul Balmer \\ Department of Mathematics \\ University of California \\ Los Angeles, CA 90095-1555 \\ balmer@math.ucla.edu \\ Robert Finn \\ Department of Mathematics \\ Stanford University \\ Stanford, CA 94305-2125 \\ finn@math.stanford.edu \\ Sorin Popa \\ Department of Mathematics \\ University of California \\ Los Angeles, CA 90095-1555 \\ popa@math.ucla.edu
}

\author{
Vyjayanthi Chari \\ Department of Mathematics \\ University of California \\ Riverside, CA 92521-0135 \\ chari@math.ucr.edu \\ Kefeng Liu \\ Department of Mathematics \\ University of California \\ Los Angeles, CA 90095-1555 \\ liu@math.ucla.edu \\ Jie Qing \\ Department of Mathematics \\ University of California \\ Santa Cruz, CA 95064 \\ qing@ cats.ucsc.edu
}

\section{PRODUCTION}

Silvio Levy, Scientific Editor, production@msp.org

\section{SUPPORTING INSTITUTIONS}

ACADEMIA SINICA, TAIPEI

CALIFORNIA INST. OF TECHNOLOGY

INST. DE MATEMÁTICA PURA E APLICADA

KEIO UNIVERSITY

MATH. SCIENCES RESEARCH INSTITUTE

NEW MEXICO STATE UNIV.

OREGON STATE UNIV.

\author{
STANFORD UNIVERSITY \\ UNIV. OF BRITISH COLUMBIA \\ UNIV. OF CALIFORNIA, BERKELEY \\ UNIV. OF CALIFORNIA, DAVIS \\ UNIV. OF CALIFORNIA, LOS ANGELES \\ UNIV. OF CALIFORNIA, RIVERSIDE \\ UNIV. OF CALIFORNIA, SAN DIEGO \\ UNIV. OF CALIF., SANTA BARBARA
}

\author{
Daryl Cooper \\ Department of Mathematics \\ University of California \\ Santa Barbara, CA 93106-3080 \\ cooper@math.ucsb.edu \\ Jiang-Hua Lu \\ Department of Mathematics \\ The University of Hong Kong \\ Pokfulam Rd., Hong Kong \\ jhlu@maths.hku.hk \\ Paul Yang \\ Department of Mathematics \\ Princeton University \\ Princeton NJ 08544-1000 \\ yang@math.princeton.edu
}

These supporting institutions contribute to the cost of publication of this Journal, but they are not owners or publishers and have no responsibility for its contents or policies.

See inside back cover or msp.org/pjm for submission instructions.

The subscription price for 2015 is US \$420/year for the electronic version, and \$570/year for print and electronic.

Subscriptions, requests for back issues and changes of subscribers address should be sent to Pacific Journal of Mathematics, P.O. Box 4163, Berkeley, CA 94704-0163, U.S.A. The Pacific Journal of Mathematics is indexed by Mathematical Reviews, Zentralblatt MATH, PASCAL CNRS Index, Referativnyi Zhurnal, Current Mathematical Publications and Web of Knowledge (Science Citation Index).

The Pacific Journal of Mathematics (ISSN 0030-8730) at the University of California, c/o Department of Mathematics, 798 Evans Hall \#3840, Berkeley, CA 94720-3840, is published twelve times a year. Periodical rate postage paid at Berkeley, CA 94704, and additional mailing offices. POSTMASTER: send address changes to Pacific Journal of Mathematics, P.O. Box 4163, Berkeley, CA 94704-0163.

PJM peer review and production are managed by EditFLOW ${ }^{\circledR}$ from Mathematical Sciences Publishers.

\section{PUBLISHED BY}

\section{mathematical sciences publishers \\ nonprofit scientific publishing}

http://msp.org/

(C) 2015 Mathematical Sciences Publishers 


\section{PACIFIC JOURNAL OF MATHEMATICS}

Volume $273 \quad$ No. $1 \quad$ January 2015

Maximal estimates for Schrödinger equations with inverse-square potential 1

ChangXing MiaO, JUNYong Zhang and JiQiang Zheng

Vassiliev Invariants of Virtual Legendrian Knots

PATRICIA CAHN and ASA LEVI

Some results on the generic vanishing of Koszul cohomology via

deformation theory

JIE WANG

Conformal metrics with constant curvature one and finitely many conical

singularities on compact Riemann surfaces

QING Chen, WeI WANG, Yingyi Wu and BIN XU

$\mathbb{Q}$-bases of the Néron-Severi groups of certain elliptic surfaces

MASAMICHI KURODA

On a prime zeta function of a graph

TAKehiro Hasegawa and SEIKEN SAIto

On Whittaker modules for a Lie algebra arising from the 2-dimensional torus 147

SHaObin TAN, QING WANG and ChENGKANG XU

Fréchet quantum supergroups

AXEL DE GOURSAC

Generators of the Gauss-Picard modular group in three complex dimensions

BaoHua Xie, JieYan Wang and YuePing Jiang

Complete characterization of isolated homogeneous hypersurface singularities

STEPHEN YAu and HuAiQING ZuO

A theorem of Mœglin and Waldspurger for covering groups

SHIV PRAKASH PATEL

Spanning trees and random walks on weighted graphs 